\title{
Non-Gaussian statistics of multiple filamentation
}

\author{
Pavel M. Lushnikov* and Natalia Vladimirova \\ Department of Mathematics and Statistics, University of New Mexico, Albuquerque, New Mexico 87131, USA \\ ${ }^{*}$ Corresponding author: plushnik@math.unm.edu
}

Received February 5, 2010; revised May 4, 2010; accepted May 11, 2010; posted May 18, 2010 (Doc. ID 123876); published June 3, 2010

\begin{abstract}
We consider the statistics of light amplitude fluctuations for the propagation of a laser beam subjected to multiple filamentation in an amplified Kerr media, with both linear and nonlinear dissipation. Dissipation arrests the catastrophic collapse of filaments, causing their disintegration into almost linear waves. These waves form a nearly Gaussian random field that seeds new filaments. For small amplitudes the probability density function (PDF) of light amplitude is close to Gaussian, while for large amplitudes the PDF has a long powerlike tail that corresponds to strong non-Gaussian fluctuations, i.e., intermittency of strong optical turbulence. This tail is determined by the universal form of near singular filaments and the PDF for the maximum amplitudes of the filaments. (c) 2010 Optical Society of America

OCIS codes: $\quad 190.0190,190.5940,260.5950$.
\end{abstract}

The self-focusing and multiple filamentation of an intense laser beam propagating through Kerr media has been the subject of intense research since the advent of lasers $[1,2]$. Multiple filamentation has many applications ranging from laser fusion [3-5] to the propagation of ultrashort pulses in the atmosphere [6,7]. Here we study the statistics of multiple filamentation, which can be viewed as an example of strong optical turbulence with intermittency [8], i.e., strong non-Gaussian fluctuations of the amplitude of the laser field [9].

Long non-Gaussian tails of the probability density function (PDF) of light amplitude fluctuations have been previously observed in filamentation experiments [10] and optical rogue waves [11]. Long tails were obtained in solutions of the complex Ginzburg-Landau equation with a quintic nonlinearity [12]. The analytical form of a long tail of PDF for velocity gradient dominated by near-singular shocks was obtained in solutions of the forced Burgers equation [13].

Here we describe the propagation of a laser beam through the amplified Kerr media by the regularized nonlinear Schrödinger equation (RNLS) in dimensionless form,

$$
i \partial_{z} \psi+(1-i a \epsilon) \nabla^{2} \psi+(1+i c \epsilon)|\psi|^{2} \psi=i \epsilon b \psi,
$$

where the beam is directed along the $z$ axis, $\mathbf{r} \equiv(x, y)$ are the transverse coordinates, $\psi(\mathbf{r}, \boldsymbol{z})$ is the envelope of the electric field, and $\nabla \equiv\left(\frac{\partial}{\partial x}, \frac{\partial}{\partial y}\right)$. The term with $a$ describes a wavenumber-dependent linear absorption, the term with $c$ corresponds to two-photon absorption, and $b$ is the linear gain coefficient. It is assumed that the wavenumber-independent part of linear absorption is included in $b$ so that the $a$-term corresponds to the expansion of the general absorption coefficient near the carrier wavenumber of the laser beam in the Fourier domain. Here, $\epsilon \ll 1$, and we generally assume $a, c, b \sim 1$. RNLS (1) is also called the complex Ginzburg-Landau equation.

RNLS (1) can be realized experimentally in numerous systems, including, e.g., the propagation of a laser beam in a ring cavity with a thin slab of Kerr media and amplification. In this case the nonlinear phase shift of the laser beam at each round trip is small, so we can obtain RNLS (1) in a mean-field approximation $[\underline{14}, \underline{15}]$ with $z$ corresponding to the number of round trips in the cavity.
RNLS (1) also describes the multiple filamentation of an intense, ultrashort laser beam in a Kerr media if we average over the temporal extent of the pulse [16]. For example, the multiple filamentation experiment in [17] with two-photon-dominated absorption corresponds to $c € \simeq 0.025$.

Neglecting dissipation and amplification, we recover the nonlinear Schrödinger equation (NLS),

$$
i \partial_{z} \psi+\nabla^{2} \psi+|\psi|^{2} \psi=0 .
$$

NLS describes a catastrophic collapse (also called wave collapse) of filaments, $\max _{\mathbf{r}}|\psi| \equiv|\psi|_{\max } \rightarrow \infty$, in a finite distance along $z$, if the optical power $N=\int|\psi|^{2} \mathrm{~d} \mathbf{r}$ is above the critical power $N_{c} \simeq 11.701$ [2].

The optical power is not conserved in RNLS (1) for $\epsilon \neq 0$. If $b>0$, the amplification term on the right-hand side of RNLS (1) results in an increase of $N$. If $b=0$ we assume that $N \gg N_{c}$ for $z=0$ (e.g., $N / N_{c} \sim 10^{4}$ in [17]). In both cases the modulational instability [2] leads to the growth of perturbations of the beam and seeds multiple collapsing filaments. These two cases are called forced and decaying turbulence, respectively, in reference to turbulence in the Navier-Stokes equations [9]. The statistical properties of these two cases are similar, provided in the decaying case we consider distances along $z$ at which a relative cumulative decay of $N$ is small.

In this Letter we focus on a forced case in which a dynamic balance is achieved between the pumping of optical power into the laser beam and dissipation. Figure 1(a) shows the evolution of $N(z)$ obtained from a numerical solution of RNLS (1). The optical power grows until reaching a statistical steady-state corresponding to fully developed optical turbulence with $N \simeq 1200$. In this regime the amplitude $|\psi|$ is characterized by the random distribution of filaments in $\mathbf{r}$ and $z$, as seen in the snapshot of $|\psi|$ for a fixed $z$ in Fig. 2. Dissipation is important only when the amplitude of each collapsing filament is near to its maximum [see Fig. 1(b)] as well as for large wavenumbers $k$. When $|\psi|_{\max }(\overline{z)}$ goes through a maximum, $N$ experiences a fast decay due to dissipation. The influence of periodic boundary conditions on the statistical properties of optical turbulence can be neglected if the simulation domain is large enough so that $N \gg N_{c}$ (in Fig. $\underline{1}, N \sim 100 N_{c}$ ). 

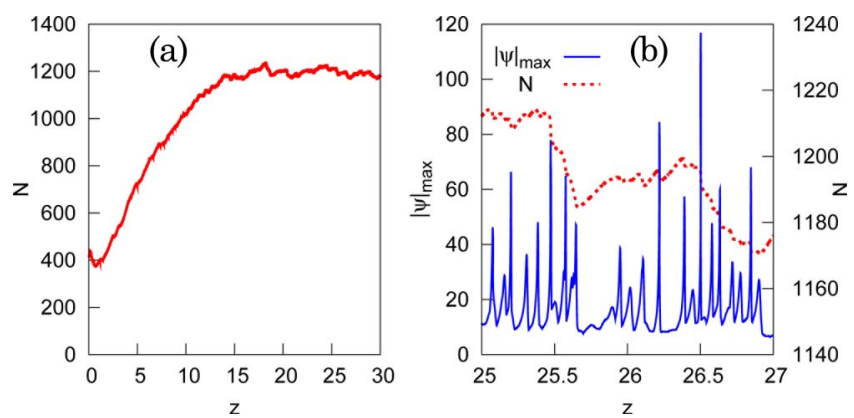

Fig. 1. (Color online) (a) $N(z)$ from simulation of RNLS (1) with $b=20, a=c=1, \epsilon=0.01$. (b) Zoom of $N(z)$ in a smaller interval in $z$ (solid curve, scale on the right) superimposed the $|\psi|_{\max }(z)$ (dashed curve, scale on the left). All simulations used the fourth-order pseudospectral split-step algorithm on $-12.8 \leq$ $x, y \leq 12.8$ with periodic boundary conditions at resolution $4096 \times 4096$ grid points. Initial conditions were a superposition of 100 randomly placed real-valued Gaussians with amplitudes and radii on $[-2,2]$ and $[1,2]$.

The evolution of each collapsing filament is well approximated for large $|\psi|$ by a self-similar radially symmetric solution $[\underline{2}, \underline{18}]$ :

$$
|\psi(\mathbf{r}, \boldsymbol{z})|=\frac{1}{L(\boldsymbol{z})} R_{0}(\rho), \quad \rho=\frac{r}{L(\boldsymbol{z})}, \quad r \equiv|\mathbf{r}|,
$$

where $L(z)$ is the transverse spatial scale of a filament and $R_{0}$ is the ground-state soliton solution of NLS (2), given by $\nabla^{2} R_{0}-R_{0}+R_{0}^{3}=0$, and corresponding to the critical power, $N_{c}=\int R_{0}(r)^{2} \mathrm{~d} \mathbf{r}[2]$.

If $\epsilon=0$ then $L(z) \simeq(2 \pi)^{1 / 2}\left(z_{0}-z\right)^{1 / 2} /\left(\ln \mid \ln \left(z_{0}-\right.\right.$ $z) \mid)^{1 / 2}$ describes a singularity (catastrophic collapse of a filament) as $z \rightarrow z_{0}[2,18]$. For $\epsilon \neq 0$ the collapse is regularized and $|\psi|_{\max }(\boldsymbol{z})$ achieves a maximum $|\psi|_{\max \max }$ at some $z=z_{\max }$. A function

$$
\gamma \equiv L \frac{\mathrm{d} L}{\mathrm{~d} z}
$$

changes slowly with $z$ compared with $L$ at $z \lesssim z_{\max }$. In the vicinity of a collapse, the forcing term in the righthand side of RNLS (1) can be neglected; the resulting equation can be written in rescaled units $z|\psi|_{\max \max }^{2}$

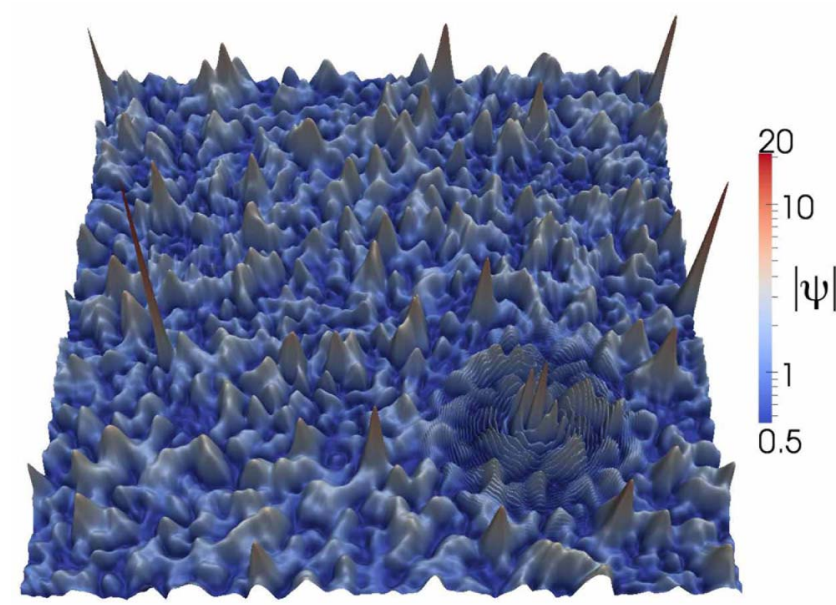

Fig. 2. (Color online) Snapshot of $|\psi|$ (vertical axis) versus spatial coordinates $(x, y)$ for simulation of Fig. 1 .
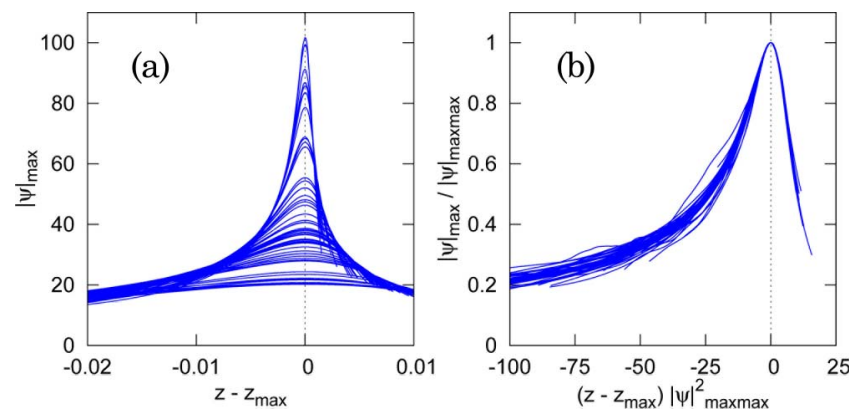

Fig. 3. (Color online) Dependence of $|\psi|_{\max }(z)$ for multiple individual collapsing filaments in (a) the nonrescaled units and (b) the rescaled units (see the text for the description of rescaling). Individual filaments are extracted from simulation of RNLS (1) with parameters of Fig. 1 .

$\mathbf{r}|\psi|_{\max \max }$, and $\psi /|\psi|_{\max \max }$. (Here, we have also shifted $z_{\max }$ to $z=0$.) As shown in Fig. $3,|\psi|_{\max }(z)$ rescaled in these units exhibits a universal behavior for all near-singular filaments, even for $\epsilon \neq 0$, and independent of the complicated structure of optical turbulence. This universality is a characteristic feature of two-photon absorption term in RNLS (1), but it may not hold for other types of absorption.

Once the amplitude of a filament reaches its maximum, the amplitude decreases and subsequently the filament decays into outgoing cylindrical waves, as seen in Fig. 2. Superposition of these almost linear waves forms a nearly random Gaussian field and seeds new filaments. Figure $\underline{4}$ shows the probability $\mathcal{P}(h)$ for the amplitude $|\psi|$ to have a value $h$, determined from simulations as

$$
\mathcal{P}(h)=\frac{\int \delta(|\psi(\mathbf{r}, z)|-h) \mathrm{d} \mathbf{r d} z}{\int \mathrm{d} \mathbf{r d} z}
$$

Here, the integrals are taken over all values of $\mathbf{r}$ and all values of $z$ after the turbulence has reached the statistically steady state. We observe that the fit to the Gaussian distribution works very well for $|\psi| \lesssim 2$, which corresponds to almost linear waves, while for $|\psi| \gtrsim 3$ the PDF has a power lawlike dependence indicating intermittency [19].
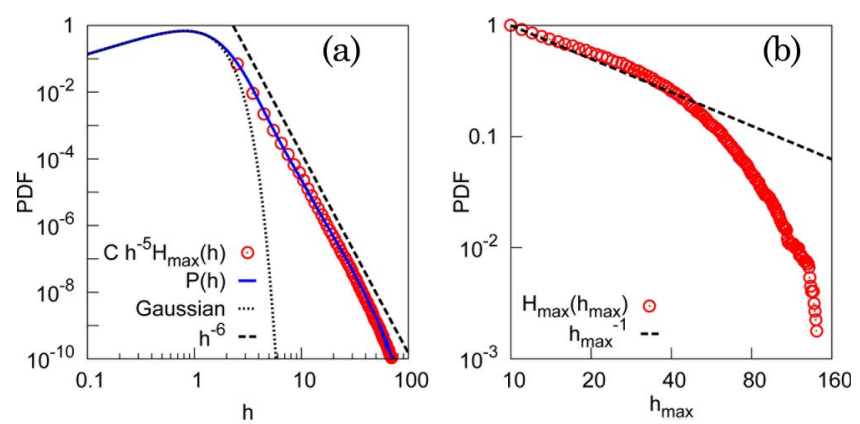

Fig. 4. (Color online) (a) $\mathcal{P}(h)$ for $|\psi|=h$ (solid curve) for the same simulation as in Fig. 1. The dashed line shows fit to the Gaussian distribution, and the dotted line shows $h^{-6}$ power law. Circles correspond to the solution of Eq. (7). (b) $H_{\max }\left(h_{\max }\right)$ for $|\psi|_{\max \max } \cdot 2231$ collapse events with $|\psi|_{\max \max }>10$ are included in simulations in log-log scale (red dots). The dotted line shows $h^{-1}$ power law. Scattering of data points for $h \gtrsim 110$ is due to lack of statistical ensemble for large collapses and is reduced for larger simulation times. 
We now show that the powerlike tail of $\mathcal{P}(h)$ results from the near-singular filaments. This approach dates back to the idea of describing strong turbulence in the Navier-Stokes equations through singularities of the Euler equations [9]. Unfortunately, this hydrodynamic problem remains unsolved. The forced Burgers equation remains the only example of an analytical description of strong turbulence in which the tail of the PDF for negative gradients follows a well-established $(-7 / 2)$ power law [13], dominated by the dynamics of near-singular shocks.

First, we calculate the contribution to the PDF from individual collapsing filaments. As shown in Fig. 3, the filament amplitude $|\psi|_{\text {max }}$ reaches the maximum $|\psi|_{\text {max } \max } \equiv$ $h_{\max }$ at $z=z_{\max }$, and rapidly decays for $z>z_{\max }$. While neglecting the contribution to $\mathcal{P}(h)$ from $z \gtrsim z_{\max }$, we calculate the contribution of an individual filament to $\mathcal{P}(h)$ through the conditional probability $\mathcal{P}\left(h \mid h_{\max }\right)$ using Eqs. (3), (5), and (ㄴ) as follows:

$$
\begin{aligned}
\mathcal{P}\left(h \mid h_{\max }\right) & \propto \int \mathrm{d} z \int \mathrm{d} \mathbf{r} \delta\left(h-\frac{1}{L(z)} R_{0}\left(\frac{r}{L(z)}\right)\right) \\
& \propto \int \mathrm{d} \rho \rho \int \frac{\mathrm{d} L L^{3}}{\gamma} \delta\left(h-\frac{1}{L(z)} R_{0}(\rho)\right) \\
& \simeq \int \frac{\mathrm{d} \rho \rho}{\langle\gamma\rangle h^{5}}\left[R_{0}(\rho)\right]^{4} \Theta\left(\frac{R_{0}(0)}{L\left(z_{\max }\right)}-h\right) \\
& =\text { Const } h^{-5} \Theta\left(h_{\max }-h\right),
\end{aligned}
$$

where $h_{\max }=R_{0}(0) / L\left(z_{\max }\right)$ and $\Theta(x)$ is the Heaviside step function. Here, we have changed the integration variable from $z$ to $L$ and approximated $\gamma(z)$ under the integral by its average value $\langle\gamma\rangle$ as $\gamma(z) \simeq\langle\gamma\rangle \sim-0$.5. This approximation is valid for $z \lesssim z_{\max }$ outside the neighborhood of $z=z_{\max }$.

As a second step we calculate $\mathcal{P}(h)$ by integration over all values of $h_{\max }$ using Eq. ( $\left.\underline{6}\right)$ as follows:

$$
\begin{aligned}
\mathcal{P}(h) & =\int \mathrm{d} h_{\max } \mathcal{P}\left(h \mid h_{\max }\right) \mathcal{P}_{\max }\left(h_{\max }\right) \\
& \simeq \text { Const } h^{-5} \int \mathrm{d} h_{\max } \Theta\left(h_{\max }-h\right) \mathcal{P}_{\max }\left(h_{\max }\right) \\
& =\text { Const } h^{-5} H_{\max }(h),
\end{aligned}
$$

where $\mathcal{P}_{\max }\left(h_{\max }\right)$ is the PDF for $h_{\max }=|\psi|_{\max \max }$ and $H_{\max }(h) \equiv \int_{h}^{\infty} \mathcal{P}_{\max }\left(h_{\max }\right) \mathrm{d} h_{\max }$ is the cumulative probability that $|\psi|_{\text {max max }}>h$.

Figure 4(b) shows $H_{\max }\left(h_{\max }\right)$. Circles in Fig. 4(a) show the prediction of Eq. ( $)$ ) with $H_{\max }\left(h_{\max }\right)$ from Fig. $4(\mathrm{~b})$.
The constant in Eq. (7) was chosen to fit the circles and the solid curve in Fig. 4(a). The very good agreement between these two curves, first, justifies the assumptions used in derivation of Eq. (7), and, second, shows that the intermittency of optical turbulence of RNLS (1) is due to collapse dynamics, which is the main result of this Letter. Figure 4 also shows that $H_{\max }\left(h_{\max }\right)$ is not well approximated by $h_{\max }^{-1}$ (and is not universal because it depends on the parameters $a, b, c$, and $\varepsilon$ ). Consequently, $h^{-6}$ is only a crude approximation for $\mathcal{P}(h)$.

Support was provided by the National Science Foundation (NSF) grant DMS 0807131 and the University of New Mexico Research Allocations Committee grant.

\section{References}

1. R. W. Boyd, Nonlinear Optics (Academic Press, 2003).

2. C. Sulem and P. L. Sulem, Nonlinear Schroedinger Equations: Self-Focusing and Wave Collapse (Springer, 1999).

3. J. D. Lindl, P. Amendt, R. L. Berger, S. G. Glendinning, S. H. Glenzer, S. W. Haan, R. L. Kauffman, O. L. Landen, and L. J. Suter, Phys. Plasmas 11, 339 (2004).

4. P. M. Lushnikov and H. A. Rose, Phys. Rev. Lett. 92, 255003 (2004).

5. P. M. Lushnikov and H. A. Rose, Plasma Phys. Controlled Fusion 48, 1501 (2006).

6. M. Mlejnek, M. Kolesik, J. V. Moloney, and E. M. Wright, Phys. Rev. Lett. 83, 2938 (1999).

7. L. Bergé, S. Skupin, R. Nuter, J. Kasparian, and J.-P. Wolf, Rep. Prog. Phys. 70, 1633 (2007).

8. S. Dyachenko, A. C. Newell, A. Pushkarev, and V. E. Zakharov, Physica D (Amsterdam) 57, 96 (1992).

9. U. Frisch, Turbulence: The Legacy of A. N. Kolmogorov (Cambridge U. Press, 1995).

10. A. Montina, U. Bortolozzo, S. Residori, and F. T. Arecchi, Phys. Rev. Lett. 103, 173901 (2009).

11. D. R. Solli, C. Ropers, P. Koonath, and B. Jalali, Nature 450, 1054 (2007).

12. H. Iwasaki and S. Toh, Progr. Theor. Phys. 87, 1127 (1992).

13. W. E, K. Khanin, A. Mazel, and Y. Sinai, Phys. Rev. Lett. 78, 1904 (1997).

14. M. Tlidi, M. Haelterman, and P. Mandel, Europhys. Lett. 42, 505 (1998).

15. P. M. Lushnikov and M. Saffman, Phys. Rev. E 62, 5793 (2000).

16. S. Tzortzakis, L. Bergé, A. Couairon, M. Franco, B. Prade, and A. Mysyrowicz, Phys. Rev. Lett. 86, 5470 (2001).

17. L. Guyon, F. Courvoisier, V. Boutou, R. Nuter, A. Vinçotte, S. Champeaux, L. Bergé, P. Glorieux, J.-P. Wolf, Phys. Rev. A 73, 051802(R) (2006).

18. G. Fibich and G. Papanicolaou, SIAM J. Appl. Math. 60, 183 (1999).

19. Y. Chung, P. M. Lushnikov, and N. Vladimirova, AIP Conf. Proc. 1168, 1235 (2009). 